\title{
Evaluación del consumo de energía metabolizable sobre el contenido energético de la canal en borregas Pelibuey
}

\section{The effects of metabolizable energy intake on carcass energy content in Pelibuey sheep}

\author{
Alfonso J. Chay-Canula ${ }^{*}$, J uan C. Ku-Vera ${ }^{b}$, Juan G. Magaña-Monforte ${ }^{b}$, Armín J . Ayala-Burgos ${ }^{b}$, \\ Ricardo A. García-Herreraa ${ }^{a}$ Angel T. Piñeiro-Vázquez ${ }^{b}$
}

\begin{abstract}
RESUMEN
El objetivo del presente estudio fue evaluar el efecto del consumo de energía metabolizable (CEM) sobre el contenido energético de la canal de ovejas Pelibuey. Veinticuatro (24) ovejas con un peso vivo de $37.2 \pm 4.0 \mathrm{~kg}$ y condición corporal de $2.5 \pm$ 0.12 se asignaron al azar a cuatro grupos de seis animales. Un grupo se sacrificó al inicio del experimento para las mediciones de referencia, y las ovejas de los grupos restantes se asignaron a uno de tres niveles de CEM: bajo (B), medio (M) y alto (A) durante 65 días. El CEM fue: $0.247,0.472$ y $0.532 \mathrm{MJ} / \mathrm{kgPV}$.75/ día para B, M y A, respectivamente. Las ovejas se sacrificaron y la canal se dividió en dos mitades; la mitad izquierda se disecó en grasa, músculo y hueso. Las ovejas en el nivel B y M removieron 55 y el $26 \%$ de su contenido de energía inicial de la canal respectivamente, mientras que el nivel A aumentó su contenido de energía en un 14 \% . Los cambios diarios en la energía en la canal fueron -1.099, -0.515 y 0.285 MJ / día para B, M y A, respectivamente. Las proporciones de la energía en canal como músculo y grasa fueron 76 y $24 \%, 68$ y $32 \%$, y 65 y $35 \%$ para los niveles de B, M y A, respectivamente. Las ovejas Pelibuey remueven aproximadamente el $55 \%$ del contenido de energía de la canal cuando se someten a condiciones de subalimentación severa.
\end{abstract}

PALABRAS CLAVE: Pelibuey, Composición de la canal, Energía de la canal, Condición corporal.

\begin{abstract}
An evaluation was done of the effect of metabolizable energy intake (MEI) on carcass energy content in Pelibuey sheep. Twenty-four (24) sheep (37.2 $\pm 4.0 \mathrm{~kg}$ live weight; $2.5 \pm 0.12$ body condition) were randomly assigned to four groups of six animals each. To establish reference data, one group was killed as the experiment began. The remaining three groups were assigned to one of three MEl levels for $65 \mathrm{~d}$ : low (L), $0.247 \mathrm{MJ} / \mathrm{kgLW}^{0.75} / \mathrm{d}$; medium (M) $0.472 \mathrm{MJ} / \mathrm{kgLW}^{0.75} / \mathrm{d}$ or high (H) $0.532 \mathrm{MJ} / \mathrm{kgLW}^{0.75} / \mathrm{d}$. After the experimental period, the animals were killed and each carcass divided longitudinally in half: the left half was dissected into fat, muscle and bone components. Carcass energy content decreased by $55 \%$ in the $L$ treatment, and by $26 \%$ in the $M$ treatment. However, it increased by $14 \%$ in the $\mathrm{H}$ treatment. Daily changes in carcass energy content were $-1.099 \mathrm{MJ} / \mathrm{d}$ in the L treatment, $-0.515 \mathrm{MJ} / \mathrm{d}$ in the $\mathrm{M}$ treatment and 0.285 in the $\mathrm{H}$ treatment. Energy stored in fat was $76 \%$ in $\mathrm{L}, 68 \%$ in $\mathrm{M}$ and $65 \%$ in $\mathrm{H}$, while energy stored in muscle was $24 \%$ in L, $32 \%$ in $\mathrm{M}$ and $35 \%$ in $\mathrm{H}$. Under conditions of severe undernourishment, Pelibuey sheep lose approximately $55 \%$ of carcass energy content.
\end{abstract}

KEY WORDS: Pelibuey sheep, Carcass composition, Carcass energy, Body condition.

Recibido el 19 de octubre de 2015. Aceptado el 13 de abril de 2016.

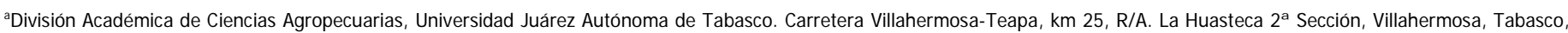
México.

bUniversidad Autónoma de Yucatán, Facultad de Medicina Veterinaria y Zootecnia, Carr. Mérida-Xmatkuil km 15.5, CP 97100 Mérida, Yucatán, México.

*Autor de correspondencia: aljuch@hotmail.com, ajchc19@yahoo.com.mx. 
En los rumiantes, la estacionalidad y el estado fisiológico resultan en fluctuaciones en el consumo de alimento, induciendo periodos alternados de subnutrición y realimentación a través del año ${ }^{(1,2)}$. Dichas variaciones estacionales inducen también periodos de pérdida y ganancia de peso en animales en pastoreo(2) así como en el peso de los órganos, del tracto gastrointestinal y la canal ${ }^{(3,4,5)}$, y en consecuencia en el contenido energético de la canal y el cuerpo. Estudios recientes han mostrado que el comportamiento en el consumo y la digestibilidad en ovinos de lana y en el ganado se afectaron cuando se utilizó la restricción alimenticia para mejorar la utilización de los nutrientes ${ }^{(6,7)}$.

También se ha reportado que la conformación y composición de la canal en ovinos de pelo puede verse afectada por factores como, el sexo y el nivel de alimentación ${ }^{(8)}$. Existe poca información en relación con la composición de la canal y los requerimientos energéticos en ovejas Pelibuey adultas ${ }^{(9,10)}$. Por otro lado, se ha reportado que el conocimiento de los requerimientos nutricionales de los animales es fundamental para incrementar la eficiencia de los sistemas de producción, siendo necesaria la determinación de la composición corporal(11,12,13).

El objetivo del presente trabajo fue evaluar el efecto del consumo de energía metabolizable (CEM) sobre la concentración de energía en los tejidos muscular y graso de ovejas Pelibuey adultas bajo condiciones tropicales.

El experimento se llevó a cabo en la Facultad de Medicina Veterinaria y Zootecnia de la Universidad Autónoma de Yucatán, México, localizada a $20^{\circ} 45^{\prime}$ $\mathrm{N}, 8^{\circ} 30^{\prime} \mathrm{W}$. El clima de la región es $\mathrm{AW}_{0}$ (cálido tropical sub-húmedo con lluvias en verano). El promedio de temperatura anual varía de 26 a 27.8 ${ }^{\circ} \mathrm{C}$, y la precipitación anual de 940 a 1,100 mm ${ }^{(14)}$.

Los animales utilizados se manejaron de acuerdo a la regulación oficial mexicana (NOM-062ZOO-1999) para el uso de animales experimentales. Veinticuatro ovejas (24) Pelibuey no gestantes y no lactantes, con peso vivo (PV) de $37.2 \pm 4.0 \mathrm{~kg} \mathrm{y}$ condición corporal (CC) de $2.5 \pm 0.12$ se asignaron aleatoriamente a cuatro grupos de seis animales. Los animales de un grupo se sacrificaron al inicio del experimento para las mediciones iniciales de los tejidos en la canal y el contenido de energía bruta. Los animales restantes se asignaron aleatoriamente a tres grupos de seis animales. Las ovejas se alojaron individualmente en jaulas metabólicas y se alimentaron con tres niveles de CEM: Bajo (L), Medio (M) y Alto (A) por 65 días, para lograr cambios en el PV. Los niveles de alimentación se establecieron como proporciones del requerimiento de EM para el mantenimiento (EMm, $0.426 \mathrm{MJ} / \mathrm{kg} \mathrm{PV} 0.75 / \mathrm{d}^{(15)}$. La dieta basal consistió de pasto Taiwán (Pennisetum purpureum) fresco, picado. Solo los tallos del pasto se utilizaron para reducir la variación de la composición nutricional a través de la fase experimental. La dieta basal se suplementó con un concentrado basado en fruto de frijol terciopelo (Mucuna pruriens, $72 \%$ en base seca [BS]), maíz molido (25 \% en BS), y melaza de caña (3 \% BS). Cada animal recibió diariamente $10 \mathrm{~g}$ de una mezcla mineral. El pasto se ofreció en dos partes iguales a las 0800 y 1500 h, a razón de 44 g MS/kg PV0.75/día, y el suplemento a una dosis de 0, 16 y $32 \mathrm{~g} \mathrm{MS} / \mathrm{kg}$ PV.75/día para los tratamientos $\mathrm{B}, \mathrm{M}$ y $\mathrm{A}$ respectivamente. La cantidad de alimento ofrecido se ajustó cada 15 días con base en el peso vivo de las ovejas. Para igualar el consumo de proteína cruda por las ovejas, una mezcla de urea y melaza de caña se les ofreció a razón de 1.8, 0.6 y $0 \mathrm{~g}$ de urea/kg PV.75/día para los tratamientos B, M y A, respectivamente.

El consumo de materia seca (MS) y la digestibilidad aparente de la MS (DMS) se determinaron en cuatro periodos de 15 días, con cinco días consecutivos de mediciones en cada periodo. Se tomaron muestras de alimento ofrecido, rechazos y excremento (10 \%) para cada animal. Al final de cada periodo se tomó una submuestra por animal, para las determinaciones de MS y cenizas. La digestibilidad de la materia orgánica (DMO) se determinó en el primer periodo de mediciones. El CEM se estimó de acuerdo al consumo de MS y la energía metabolizable de la dieta se estimó utilizando la MO digestible en la MS (MODMS) por medio de la ecuación $\mathbf{1}^{(15)}$.

$$
\text { EM de la dieta }(\mathrm{MJ} / \mathrm{kg} \mathrm{MS})=\% \text { MODMS } \times 0.16
$$

El PV de las ovejas se registró cada 15 días previo ayuno de alimento y agua por $18 \mathrm{~h}$. El cambio diario de PV (CDPV) se estimó por regresión utilizando los registros del PV, la pendiente de la regresión representó la tasa de CDPV en las ovejas. 
Al final del periodo de mediciones, las ovejas se sacrificaron humanitariamente de acuerdo a las normas oficiales mexicanas (NOM-08-ZOO, NOM-09ZOO y NOM-033-ZOO) establecidas para el procesamiento de animales para producción de carne. El PV en ayuno (PVA) se midió como el PV después del ayuno de alimento y agua por $24 \mathrm{~h}$. Los datos registrados fueron peso de las vísceras, canal y grasa interna (tejido adiposo interno). El tracto gastrointestinal (TGI) se pesó antes y después de ser vaciado. El PV vacío (PW) se calculó como la diferencia entre el PVA al sacrificio y el contenido del TGl . La canal se dividió por la línea media dorsal en dos mitades, pesada y refrigerada a $6{ }^{\circ} \mathrm{C}$ durante 24 h. Después de la refrigeración, la canal se pesó y la media canal izquierda fue completamente disecada en grasa (subcutánea e intermuscular), músculo, hueso, y cada componente se pesó por separado. El peso de los tejidos disecados en la media canal izquierda se ajustó a la canal completa.

Los tejidos muscular y adiposo se molieron en forma separada en un molino para carne (Torrey, México) con una criba de $4 \mathrm{~mm}$ y se tomó una muestra (1 kg) de cada animal, y se almacenó a $-20{ }^{\circ} \mathrm{C}$ en contenedores de plástico para sus análisis químicos.

Las muestras de alimento y excremento se secaron en una estufa de aire forzado a $60{ }^{\circ} \mathrm{C}$ por $48 \mathrm{~h}$ para la determinación de MS. Las muestras secas se molieron en un molino Wiley con una criba de $1 \mathrm{~mm}$ para sus análisis químicos. La MO se determinó por incineración de la muestra en una mufla a $600{ }^{\circ} \mathrm{C}$ por $6 \mathrm{~h}$. La fibra detergente neutra (FDN) y la fibra detergente ácida (FDA) se determinaron utilizando los métodos descritos por Van Soest et a/(16).

Las muestras de tejido (músculo y tejido adiposos) se liofilizaron para determinar el contenido de agua (Labconco, Co., USA). Las muestras secas se molieron en un molino de martillos para sus determinaciones químicas. Los análisis químicos realizados fueron energía bruta en una bomba adiabática (Parr, USA), cenizas, y extracto etéreo ${ }^{(17)}$ del tejido muscular y graso. La materia orgánica libre de grasa (MOLG) se calculó para ambos tejidos por diferencia y fue considerada como el contenido de proteína de los tejidos ${ }^{(18)}$.

Al final del experimento, una oveja de tratamiento B y otra del tratamiento A se retiraron del experimento a causa de enfermedad y sus datos no se incluyeron en el análisis. Los datos se analizaron como un diseño completamente al azar, utilizando el análisis de varianza y la prueba de Tukey, cuando fueron detectadas diferencias significativas entre los tratamientos. Las pruebas estadísticas se realizaron con el PROC GLM de SAS ${ }^{(19)}$.

La composición química del forraje se presenta en el Cuadro 1. El forraje presentó un bajo contenido de proteína cruda y alto en FDN comparado con el suplemento. El consumo total de MS fue diferente

Cuadro 1. Composición química del forraje y el suplemento ofrecido a ovejas Pelibuey adultas, no gestantes y no lactantes (g/kg MS)

\begin{tabular}{lcc}
\hline & Suplemento & Forraje \\
\hline MS & 900 & 283 \\
PC & 141 & 31 \\
FDN & 425 & 693 \\
FDA & 180 & 470 \\
MO & 963 & 953 \\
EE & 37 & 19.2 \\
EM, MJ/kg MS* & 11.5 & 7.6 \\
\hline
\end{tabular}

*Estimada de AFRC(15)

$\mathrm{MS}=$ materia seca; $\mathrm{PC}=$ proteína cruda; FDN= fibra detergente neutra; FDA= fibra detergente ácida; $\mathrm{MO}=$ materia orgánica; $\mathrm{EE}=$ extracto etéreo; $\mathrm{EM}=$ energía metabolizable.

Cuadro 2. Consumo de materia seca (MS) y energía metabolizable (CEM) en ovejas Pelibuey adultas, no gestantes y no lactantes alimentadas con tres niveles de consumo de energía metabolizable

\begin{tabular}{lccccc}
\hline & Bajo $(\mathrm{n}=5)$ & Medio $(\mathrm{n}=6)$ & Alto $(\mathrm{n}=5)$ & EEM & $P$ \\
\cline { 2 - 6 } Total MS, g/d & $504.6^{\mathrm{a}}$ & $815.9^{\mathrm{b}}$ & $1045.2^{\mathrm{c}}$ & 31.83 & $<0.0001$ \\
Total MS, g/kg0.75/d & $36.1^{\mathrm{a}}$ & $56.2^{\mathrm{b}}$ & $65.7^{\mathrm{c}}$ & 1.336 & $<0.0001$ \\
${ }^{*}$ CEM en la dieta, MJ/kg MS & $7.6^{\mathrm{a}}$ & $8.7^{\mathrm{a}}$ & $8.9^{\mathrm{a}}$ & 0.211 & 0.0564 \\
CEM, MJ/d & $3.40^{\mathrm{a}}$ & $7.09^{\mathrm{b}}$ & $9.29^{\mathrm{c}}$ & 0.287 & $<0.0001$ \\
CEM, MJ/kg0.75/d & $0.247^{\mathrm{a}}$ & $0.472^{\mathrm{b}}$ & $0.532^{\mathrm{b}}$ & 0.019 & 0.062 \\
\hline
\end{tabular}

* Estimada por digestibilidad de la materia orgánica de la materia seca $\times 0.16^{(15)} \mathrm{EEM}=$ error estándar de la media.

abc Valores con diferente literal difieren significativamente. 
$(P<0.05)$ entre tratamientos (Cuadro 2). El CEM estimado fue 58,110 y $125 \%$ de los requerimientos de EM para el mantenimiento (EMm: $0.426 \mathrm{MJ}$ $\mathrm{EM} / \mathrm{kg} \mathrm{PV}^{0.75}$ ) para B, M y A respectivamente ${ }^{(20)}$. El CEM promedio durante el experimento fue 0.247 , 0.472 y $0.532 \mathrm{MJ} / \mathrm{kg} \mathrm{PV}$. $75 /$ día para los niveles $\mathrm{B}, \mathrm{M}$ y A respectivamente ${ }^{(20)}$. El cambio diario de peso en las ovejas Pelibuey adultas fue de $-106,-22$ y 30

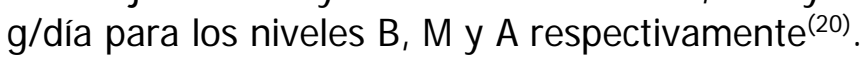

Los pesos de la canal fría y caliente fueron superiores para el nivel $\mathrm{A}$ con relación al $\mathrm{B}(P<0.05)$, sin embargo, estos no difirieron del nivel de alimentación M (Cuadro 3). El peso y las proporciones del músculo fueron superiores para $\mathrm{A}$ con respecto a $B$, sin embargo, estos no fueron diferentes del grupo M $(P<0.05)$. El peso y la proporción de la grasa de la canal fue diferente entre los grupos de alimentación $(P<0.05)$.

El Cuadro 4 presenta la composición química del tejido muscular y adiposo de la canal de ovejas Pelibuey adultas. El contenido de agua en el tejido muscular fue superior en $B$ respecto al grupo de alimentación $\mathrm{A}(P<0.001)$, sin embargo, los grupos $B$ y $M$ fueron similares $(P>0.05)$. En el tejido adiposo de la canal, el contenido de agua en el nivel $\mathrm{B}$ fue bajo $(P<0.001)$ con respecto a los grupos de

Cuadro 3. Pesos y características de la canal de ovejas Pelibuey secas alimentadas con tres niveles de consumo de energía metabolizable

\begin{tabular}{|c|c|c|c|c|c|c|}
\hline & $\begin{array}{l}\text { Inicial } \\
(n=6)\end{array}$ & $\begin{array}{l}\text { Bajo } \\
(n=5)\end{array}$ & $\begin{array}{l}\text { Medio } \\
(\mathrm{n}=6)\end{array}$ & $\begin{array}{c}\text { Alto } \\
(n=5)\end{array}$ & EEM & $P$ \\
\hline \multicolumn{7}{|l|}{ Peso corporal (kg): } \\
\hline Inicial & 36.5 & 38.2 & 36.2 & 38.7 & 1.036 & 0.0667 \\
\hline Final & - & $30.2^{a}$ & $34.8 \mathrm{ab}$ & $40.8^{b}$ & 0.988 & 0.0022 \\
\hline Ganancia diaria de peso, g/d & - & $-106^{a}$ & $-22 b$ & $30^{c}$ & 4.811 & $<0.0001$ \\
\hline \multicolumn{7}{|l|}{ Componentes canal (kg): } \\
\hline Peso canal caliente & 15.13 & $12.4^{\mathrm{a}}$ & $14.8 \mathrm{ab}$ & $17.5^{b}$ & 0.443 & 0.0022 \\
\hline Peso canal fría & 15.03 & $11.2^{a}$ & $14.3 \mathrm{ab}$ & $17.0 \mathrm{~b}$ & 0.478 & 0.0030 \\
\hline \multicolumn{7}{|l|}{ Tejidos en canal $(\mathrm{kg})$ : } \\
\hline Músculo & 8.80 & $7.17^{a}$ & $9.13^{b}$ & $10.68 \mathrm{~b}$ & 0.139 & 0.0006 \\
\hline Grasa & 2.34 & $0.81^{a}$ & $1.47^{\mathrm{b}}$ & $2.17^{c}$ & 0.046 & 0.0002 \\
\hline Hueso & 3.92 & 3.82 & 3.79 & 4.22 & 0.044 & 0.0756 \\
\hline
\end{tabular}

abc Valores con diferente literal difieren significativamente.

Cuadro 4. Composición química del tejido muscular y graso de la canal de ovejas Pelibuey ( $\mathrm{g} / \mathrm{kg}$ en base fresca) alimentadas con tres niveles de consumo de energía metabolizable

\begin{tabular}{|c|c|c|c|c|c|c|}
\hline & Inicial $(n=6)$ & Bajo $(n=5)$ & Mediano $(n=6)$ & Alto $(n=5)$ & EEM & $P$ \\
\hline \multicolumn{7}{|l|}{ Músculo: } \\
\hline Agua & 701.5 & $767.0^{\mathrm{a}}$ & $737.7^{a}$ & $687.8^{\mathrm{b}}$ & 5.47 & 0.0002 \\
\hline Grasa & 182.1 & $61.7 \mathrm{a}$ & 84.9 ab & $117.5^{b}$ & 6.06 & 0.0100 \\
\hline MOLG & 85.6 & $136.2^{a}$ & 145.6 ab & $164.3^{b}$ & 3.36 & 0.0172 \\
\hline Cenizas & 29.7 & 35.1 & 31.8 & 30.5 & 0.775 & 0.0866 \\
\hline \multicolumn{7}{|c|}{ Tejido adiposo: } \\
\hline Agua & 357.5 & $515.3^{a}$ & $423.9 \mathrm{~b}$ & $350.7^{b}$ & 12.44 & 0.0006 \\
\hline Grasa & 566.2 & 354.6 a & $493.2 \mathrm{~b}$ & $606.4^{c}$ & 17.02 & 0.0002 \\
\hline MOLG & 69.0 & $117.3^{a}$ & $73.7^{b}$ & $37.3^{c}$ & 5.21 & 0.0002 \\
\hline Cenizas & 7.3 & $12.7^{\mathrm{a}}$ & $9.1 \mathrm{ab}$ & $5.6^{b}$ & 0.592 & 0.0015 \\
\hline
\end{tabular}

MOLG= materia orgánica libre de grasa.

abc Valores con diferente literal difieren sianificativamente. 
alimentación M y A, pero estos no difirieron entre sí ( $P>0.05$ ). Como se esperaba, el contenido de grasa en el tejido adiposo fue superior al del tejido muscular; el contenido de grasa en el tejido muscular fue mayor en el grupo A con respecto al grupo B $(P<0.05)$; sin embargo, el grupo $M$ fue similar a ambos niveles. En relación con el contenido de MOLG, en el músculo, el grupo B fue superior al nivel $\mathrm{A}(P<0.01)$, no obstante, el grupo $\mathrm{M}$ fue similar a ambos grupos; en el tejido adiposo, el contenido de MOLG fue mayor en el nivel $\mathrm{B}(P<0.001)$. Sobre el contenido de cenizas en el tejido muscular, no hubo diferencias entre tratamientos, aunque en el tejido adiposo, el contenido de cenizas en nivel $B$ fue mayor respecto al grupo $\mathrm{A}(P<0.01)$.

Se encontraron diferencias $(P<0.05)$ en el peso del músculo (BS) entre el nivel de alimentación A con respecto a $\mathrm{M}$ y $\mathrm{B}$, sin embargo, estos últimos fueron similares. Asimismo, se encontraron diferencias $(P<0.05)$ para el peso de la grasa (BS) entre los niveles de alimentación. La concentración de energía en el tejido muscular en la canal presentó diferencias $(P<0.05)$ entre los niveles de alimentación. Para la energía contenida como grasa en la canal y el contenido total de energía en la canal, se encontró que el nivel $A$ fue superior a los niveles $M$ y $B$ $(P<0.05)$, sin embargo, estos últimos fueron similares entre sí (Cuadro 5). Las proporciones de energía contenida como músculo y grasa fueron 76 y $24 \%, 68$ y $32 \%$, y 65 y $35 \%$ para los niveles de alimentación $\mathrm{B}, \mathrm{M}$ y $\mathrm{A}$ respectivamente. Por otro lado, los cambios diarios en promedio de energía en el cuerpo (estimada como el contenido de energía en la canal del grupo inicial menos el contenido final de la energía en la canal de los otros grupos y dividida entre los días en experimentación), fueron $1.099,-0.515$ y $0.285 \mathrm{MJ}$ para los niveles B, M y A respectivamente. Los resultados obtenidos indican que las ovejas alimentadas con el nivel $\mathrm{B}$ removieron de la canal alrededor del $55 \%$ de la energía inicial, también, en el nivel $M$ removieron cerca del $26 \%$ con respecto a la energía inicial en 65 días. Por el contrario el grupo A aumentó el contenido de energía de la canal en un 14 \% en comparación con el contenido de energía inicial.

El peso de la canal de las ovejas se incrementó con el nivel de alimentación, lo cual está de acuerdo con lo reportado por otros autores ${ }^{(21-25)}$. En este respecto, se ha encontrado que el peso de la canal es bajo en animales subalimentados ${ }^{(4)}$. Atti et $a{ }^{(4)}$, Kamalzadeh et $a{ }^{(22)}$ y Aziz et $a{ }^{(21)}$ reportaron que el rendimiento de la canal y el peso fueron bajos en animales que fueron subalimentados; esto debido a un incremento en las proporciones de los desperdicios y también a la movilización de la grasa.

Los valores para el porcentaje de músculo en la canal de ovinos Pelibuey mencionados en una investigación ${ }^{(25)}$, fueron superiores a los reportados en el presente trabajo. El porcentaje de hueso reportado por estos autores fue comparable con el del nivel $B$. El peso de la grasa en la canal fue diferente entre los tratamientos $(P<0.05)$ y presentó una respuesta lineal al CEM, lo que está de acuerdo con lo reportado para animales en crecimiento recibiendo tres niveles de alimentación ${ }^{(22,23)}$. El peso del hueso fue similar entre tratamientos ( $P>0.05$ ); en este contexto, se ha reportado que en animales en crecimiento el peso del hueso no es afectado por

Cuadro 5. Contenido de MS $(\mathrm{kg})$ y energía bruta $(\mathrm{MJ})$ del tejido muscular y graso de la canal de ovejas Pelibuey alimentadas con tres niveles de consumo de energía metabolizable

\begin{tabular}{|c|c|c|c|c|c|c|}
\hline & Inicial $(n=6)$ & Bajo $(n=5)$ & Medio $(n=6)$ & Alto $(n=5)$ & EEM & $P$ \\
\hline \multicolumn{7}{|c|}{ Tejidos (kg MS): } \\
\hline Músculo & 2.65 & $1.67^{a}$ & $2.39 \mathrm{a}$ & $3.36^{b}$ & 0.132 & 0.0008 \\
\hline Grasa & 1.50 & $0.39 a$ & $0.86^{b}$ & $1.41^{c}$ & 0.067 & 0.0002 \\
\hline \multicolumn{7}{|c|}{ Energía bruta (MJ): } \\
\hline Músculo & 74.13 & 43.89 a & $65.07^{a}$ & $95.25^{b}$ & 4.56 & 0.0023 \\
\hline Grasa canal & 54.92 & $13.73^{a}$ & $30.54^{b}$ & $52.33^{c}$ & 2.49 & 0.0001 \\
\hline Total canal & 129.05 & $57.62^{a}$ & $95.60^{a}$ & 147.592 b & 6.65 & 0.0005 \\
\hline
\end{tabular}

abc Valores con diferente literal difieren significativamente. 
el nivel de alimentación, esto es debido al hecho de que el hueso es un tejido de maduración temprana, que es más una función de la edad del animal que del nivel de alimentación a que está siendo sometido(22,23).

Respecto a la composición y contenido energético de la canal, Peraza-Mercado et a/26) encontraron que el músculo $L$. dorsi de corderos Pelibuey tuvo un contenido de humedad de $73 \%$, cenizas de $1.4 \%$, grasa cruda de $4.5 \%$ y proteína cruda de $16.4 \%$. Estos valores son similares a los registrados en el presente estudio (Cuadro 2). Resultados similares fueron reportados en otras investigaciones ${ }^{(27)}$, donde registraron $74 \%$ de humedad, $4 \%$ de grasa, $15.3 \%$ de proteína cruda y $1.4 \%$ de cenizas en el músculo $L$. dorsi de corderos Pelibuey. Hernández-Cruz et $a^{(28)}$ en la carne de ovinos de pelo encontraron que la humedad fue de $74 \%$, la proteína cruda varió de 20 a $21 \%$ y las cenizas fueron de $0.8 \%$. En otro trabajo ${ }^{(29)}$ se reportan valores de $75 \%$ de humedad, $19.5 \%$ de proteína cruda, $4.5 \%$ de grasa y $1 \%$ de cenizas en el músculo Longissimus lumborum de la canal de ovejas de desecho. En este contexto, Resende et $a^{(30)}$ encontraron que en ovinos Santa I nês la composición corporal fue: 64 a $68 \%$ agua, 14 a $17 \%$ proteína cruda y 8 a $18 \%$ de grasa, en animales con pesos entre 5 y $45 \mathrm{~kg}$ de PV. En corderos Morada Nova con peso de 5 a $25 \mathrm{~kg}$, se menciona ${ }^{(31)}$ que la composición corporal varió de 64 a $70 \%$ agua, 17.8 a $18.1 \%$ proteína cruda y 6.7 a $12.1 \%$ de grasa, valores similares a los reportados en el presente estudio.

Shadnoush et $a^{(32)}$ no encontraron efecto del nivel de CEM sobre el contenido de MS, PC y cenizas en la canal de corderos machos Lori-Bakhtiari. Otros investigadores(33) encontraron que el nivel de energía metabolizable en la dieta afectó el contenido de humedad y proteína cruda de la carne de corderos; sin embargo, la grasa y cenizas no se afectaron. Por el contrario, se menciona ${ }^{(34)}$ que los porcentajes de agua y grasa en la canal de ovinos y cabras sometidos a diferentes niveles de CEM se afectaron significativamente por la densidad energética de la dieta. Además, Fozooni y Zamiri(35) no encontraron efecto de la raza, nivel de alimentación y su interacción sobre la composición de la carne de varios cortes y de la canal completa. Estos autores reportaron para la canal completa, valores promedio de $39 \%$ MS, $38.6 \%$ PC (\% MS); $55 \%$ grasa (\% MS) y $1.9 \%$ cenizas (\% MS). No se han reportado estudios donde los tejidos fueron evaluados separadamente de la canal de ovinos y menos aún, de la canal de ovejas adultas.

Las concentraciones de energía bruta en la canal de las ovejas fueron superiores a las reportados en otro trabajo con ovinos de pelo en crecimiento con pesos de 30 a $35 \mathrm{~kg}^{(36)}$; estos autores estimaron el valor del contenido de energía en la canal (por medio del peso del músculo y la grasa en la canal) entre 53.8 y $88.0 \mathrm{MJ}$.

Se concluye que las ovejas Pelibuey remueven aproximadamente el $55 \%$ del contenido energético de la canal cuando son sometidas a condiciones de subalimentación severa.

\section{LITERATURA CITADA}

1. Chilliard Y, F Bocquier, M Doreau. Digestive and metabolic adaptations of ruminants to undernutrition, and consequences on reproduction. A review. Reprod Nutr Dev 1998;38:129-150.

2. Kamalzadeh A, Koops WJ, Kiasat A. Effect of qualitative feed restriction on energy metabolism and nitrogen retention in sheep. S Afr J Anim 2009;39:30-39.

3. Aziz NN, Murray DM, Ball RA. The effect of live weight gain and live weight loss on body composition of Merino wethers: non carcass organs. J Anim Sci 1993; 71:400-407.

4. Atti N, Nozière $P$, Doreau $M$, Kayouli $C$, Bocquier F. Effects of underfeeding and refeeding on offals weight in the Barbary ewes. Small Ruminant Res 2000; 38:37-43.

5. Mahouachi M, Atti N. Effects of restricted feeding and re-feeding of Barbarine lams: intake, growth and non-carcass components. Anim Sci 2005;81:305-312.

6. Dias RS, Patino HO, López S, Prates E, Swanson KC, France J. Relationships between chewing behavior, digestibility, and digesta passage kinetics in steers fed oat hay at restricted and ad libitum intakes. J Anim Sci 2011;89: 1873-1880.

7. Souza-Rodrigues RT, Chizzotti ML, Rodrigues-Martins S, ÁvilaQueiroz MA, Costa-Busato K. Digestibility, ingestive behaviour and performance of non-descript breed hair lambs of different sexual classes subjected to feed restriction. J Anim Feed Sci 2014;23:117123.

8. Partida PJA, Rojas LM. Composición corporal de corderos Pelibuey en función de la concentración energética de la dieta y del peso al sacrificio [Body composition in Pelibuey lambs in terms of feed energy concentration and slaughter weight]. Vet Méx 2010;41:117-190.

9. Cantón GJ , Moguel Y, Castellanos A. Estimación del requerimiento energético de mantenimiento del borrego Pelibuey en clima tropical. Tec Pecu Mex 1995;33:66-73. 
10. Duarte VF. Estimación de los requerimientos energéticos del borrego Pelibuey en crecimiento para la adecuación del modelo CNCPS/SRNS [tesis doctorado]. Universidad Autónoma de Yucatán. México; 2007.

11. Scholz AM, Bünger L, Kongsro J, Baulain U, Mitchell AD. Noninvasive methods for the determination of body and carcass composition in livestock: dual-energy X-ray absorptiometry, computed tomography, magnetic resonance imaging and ultrasound: invited review. Animal 2015; (7):1250-1264.

12. Silva TS, Chizzotti ML, Busato KC, de Souza Rodrigues RT, da Silva IF, \& Queiroz MAÁ. Indirect methods for predicting body composition of Boer crossbreds and indigenous goats from the Brazilian semiarid. Trop Anim Health Prod 2015; (6):1-4.

13. Chay-Canul AJ, Magaña-Monforte JG, Chizzotti ML, Piñeiro-Vázquez AT, Canul-Solís J R, Ayala-Burgos AJ, Ku-Vera JC, et al. Energy requirements of hair sheep in the tropical regions of Latin America. Review. Rev Mex Cienc Pecu 2016; (1): 105-125.

14. García E. Modificaciones del sistema de clasificación climática de Köppen (para adaptarlo a las condiciones de la República Mexicana). Instituto de Geografía, UNAM, México, DF; 1988.

15. AFRC. Technical Committee on Responses to Nutrients. Energy and protein requirements of ruminants. CAB International, Wallingford, UK; 1993.

16. Van Soest PJ, Robertson JB, Lewis BA. Methods for dietary fiber, neutral detergent fiber and non-starch polysaccharides in relation to animal nutrition. J Dairy Sci 1991;74:3583-3597.

17. AOAC. Official Methods of Analysis. Association of Official Analytical Chemists. 15 th ed. Washington DC, USA; 1980.

18. Olthoff JC, Dickerson GE. Composition of the whole body and component fractions in mature ewes from seven breeds. J Anim Sci 1989; 67:2565-2575.

19. SAS. Institute Inc., SAS/STAT. Software, Ver. 9.00, Cary, NC275128000. USA; 2002.

20. Chay-Canul AJ, Ayala-Burgos AJ, Kú-Vera J C, Magaña-Monforte JG, Ferrell CL. Metabolizable energy intake and changes in body weight and body condition of Pelibuey ewes fed three levels of roughage diets under tropical conditions. Trop Subtrop Agroecosyst 2011; 14:777-786.

21. Aziz NN, Murray DM, Ball RA. The effect of live weight gain and live weight loss on body composition of Merino wethers: dissected muscle, fat and bone. J Anim Sci 1993; 70: 1819-1828.

22. Kamalzadeh A, Koops WJ, van Bruchem J, Tammimga S, Zward D. Feed quality restriction and compensatory growth in growing sheep: development of body organs. Small Ruminant Res 1998;29:71-82.

23. Atti $\mathrm{N}$, Rouissi $\mathrm{H}$, Mahouachi $\mathrm{M}$. Effects of restricted feeding and refeeding of Barbarine lams: carcass composition and fatty acid composition. J Anim Sci 2005;81:313-318.
24. Alves $K$, Carvalho F, Ferreira M, Véras A, Medeiros A, Nascimento $\mathrm{J}$, et al. Níveis de energia em dietas para ovinos Santa Inês: Características de carcaça e constituintes corporais. R Bras Zoot 2003; 32: 1927-1936.

25. Martínez A, Bores R, Castellanos A. Zoometría y predicción de la composición corporal de la borrega Pelibuey. Téc Pecu Méx 1987:25:72-84.

26. Peraza-Mercado G, Jaramillo-López E, Alarcón-Rojo AD. Breed effect upon carcass characteristiscs and meat quality of Pelibuey and Polipay $\times$ Ramboullet lambs. Am-Euras. J Agric \& Environ Sci 2010;8:508-513.

27. Peraza-Mercado G, J aramillo-López E, Chávez del Hierro S, AlarcónRojo AD. Diet effect upon chemical composition of Pelibuey and Polipay $\times$ Rambouillet meat. Am-Euras J Sci Res 2006;(1):8-11.

28. Hernández-Cruz L, Ramírez-Bribiesca JE, Guerrero-Legarreta MI, Hernández-Mendo $\mathrm{O}$, Crosby-Galvan MM, Hernández-Calva LM. Effects of crossbreeding on carcass and meat quality of Mexican lambs. Arq Bras Med Vet Zoot 2009;61:475-483.

29. Pinheiro RSB. Características de caraca e de carne de ovelhas Santa Ines abatidas em tres estágios fisiológicos. [tesis doctorado] Universidad Estadual Pauslista: Botacu, Brazil; 2009.

30. Resende KT, MHM Fernandes, IAMA Teixeira. Exigências nutricionais de ovinos. In: Reunião Anual da Sociedade Brasileira de Zootecnia, 42, Goiânia. Anais. Goiânia: SBZ/UFG; 2005: 114-135.

31. Gonzaga-Neto S. Composição corporal, exigências nutricionais e características da carcaça de cordeiros Morada Nova. [tesis doctorado]. Universidade Estadual Paulista, Jaboticabal, Brazil; 2003.

32. Shadnoush GH, Ghorbani GR, Edris MA. Effect of different energy levels in feed and slaughter weights on carcass and chemical composition of Lori-Bakhtiari ram lambs. Small Ruminant Res 2004;51:243-249.

33. Batista ASM, Costa RG, Garruti D dos S, Madruga MS, Queiroga RCR do $E$, Filho JT de A. Effect of energy concentration in the diets on sensorial and chemical parameters of Morada Nova, Santa Inez and Santa I nez × Dorper lamb meat. R Bras Zoot 2010;39:2017-2023.

34. Mahgoub O, Lu CD. Influence of various levels of metabolizable energy on chemical composition of whole carcass and non-carcass portion of goats and sheep. S Afr J Anim 2004;34:81-84.

35. Fozooni R, Zamiri MJ . Relationships between chemical composition of meat from carcass cuts and the whole carcass in I ranian fattailed sheep as affected by breed and feeding level. Iran J Vet Res 2007; 8:304-312.

36. Cantón C G, Bores QR, Baeza RJ, Quintal FJ, Santos RR, Sandoval CC. Energy retention of F1 Pelibuey lambs with breeds for meat production. J Anim Vet Adv 2009;8:2655-2661. 